\title{
A Programmable Differential Transimpedance Amplifier for Capacitive MEMS Accelerometers
}

\author{
Guillermo Royo ${ }^{1}$, Cecilia Gimeno ${ }^{2}$, Concepción Aldea ${ }^{1}$, Santiago Celma ${ }^{1}$ \\ ${ }^{1}$ Grupo de Diseño Electrónico (GDE) \\ Instituto de Investigación en Ingeniería de Aragón (I3A). \\ Universidad de Zaragoza, Mariano Esquillor s/n, 50018, Zaragoza, Spain. \\ Tel.+34-976762707, e-mail: royo@unizar.es \\ ${ }^{2}$ ICTEAM Institute, Université Catholique de Louvain
}

\begin{abstract}
In this work, a fully-differential transimpedance amplifier with programmable gain and bandwidth for MEMS accelerometers is presented. It is aimed for a differential surface-micromachined combfinger capacitive accelerometer, but can be used in many other capacitive sensor applications.
\end{abstract}

\section{Introduction}

MEMS capacitive sensors have become very popular over the last years, since they offer excellent noise performance, low power consumption, and a huge range of applications, from the automotive industry to videogames and electronic devices such as smartphones or tablets. One of the main uses of these sensors is the capacitive accelerometer. As shown in Fig. 1, the acceleration causes a displacement of a proof mass that produces a small change in the capacitance between it and fixed electrodes [1]. This displacement is measured by stimulating the sensor with an ac voltage signal $V_{c}$. If the frequency of the carrier signal is much higher than the capacitance variations and the mechanical resonance, the current through the capacitors can be approximated by:

$$
\begin{aligned}
& i_{1}=\left[C_{0}+\Delta C(t)\right] \frac{\partial V_{C}}{\partial t} \\
& i_{2}=\left[C_{0}-\Delta C(t)\right] \frac{\partial V_{C}}{\partial t}
\end{aligned}
$$

where $C_{0}$ is the nominal capacitance and the capaticance variation $C$, which is proportional to the displacement, can be either constant due to a static acceleration, or varying at frequencies up to a few kilohertzs. Thereby, the carrier signal is modulated by the variations of the capacitance. In order to measure the acceleration from the change in the capacitance, several techniques based on synchronous demodulation have been developed [2]. However, as the changes in the capacitance tend to be extremely small, a highly-linear, low-noise sensor interface is needed. In this work, a differential capacitive bridge has been employed, achieving power supply rejection and cancellation of substrate coupling. In order to operate with a linear displacement-to-voltage behavior and minimize the effects of the parasitic capacitances, a differential transimpedance amplifier (TIA) is proposed. The TIA converts the difference between $i_{1}$ and $i_{2}$ to a measurable voltage signal, which is then synchronously demodulated and filtered to obtain a signal proportional to the capacitance change, therefore allowing the measurement of the acceleration.

\section{Circuit description}

The proposed sensor interface shown in Fig. 2, consists of three main stages: (i) a transimpedance amplifier; (ii) a demodulator; and (iii) a low-pass filter.

This work focuses on the design of the TIA, which is the first and most critical stage in terms of bandwidth, noise and linearity [3]. The carrier frequency that stimulates the sensor depends on the application and the inertial MEMS. In order to optimize the performance of the interface, the bandwidth of the TIA must be adapted to the carrier frequency of each application. If the bandwidth of the TIA is close to the carrier frequency, the signal at the output is not synchronized with the input, and it should be demodulated with a phase-shifted replica of the carrier signal, in order to maximize the sensitivity, which requires a more complex interface. Moreover, as there is a tradeoff between gain, bandwidth and noise, adjusting the gain and bandwidth of the TIA also optimizes the noise performance, allowing the measurement of even smaller accelerations. In this work, we propose a new high gain differential voltage amplifier, implemented, as shown in Fig. 3, with a three 
cascaded differential pairs with load resistors in the first two pairs, and a resistive feedback, in a shuntshunt topology, which presents low impedance input, high linearity and low noise.

\section{Results}

The transimpedance amplifier has been designed in an 180-nm CMOS technology and it is aimed for a capacitive sensor with capacitance up to $1 \mathrm{pF}$. To carry out the simulations, a $1 \mathrm{pF}$ parasitic capacitance, $C_{P}$, has been modeled. As shown in Fig. 4, the TIA performs programmable bandwidth from $50 \mathrm{kHz}$ up to $1 \mathrm{MHz}$, as well as the transimpedance can be modified while keeping a constant bandwidth. At the maximum transimpedance configuration, the TIA presents a minimum equivalent input noise of only $45 \mathrm{fA} / \sqrt{\mathrm{Hz}}$, that corresponds to a capacitive resolution of 0.02 $\mathrm{aF} / \sqrt{\mathrm{Hz}}$ at $50 \mathrm{kHz}$. With a power consumption of only $54 \mu \mathrm{W}$, it achieves a maximum sensitivity of 1 $\mathrm{mV} / \mathrm{fF}$, or equivalently $3 \mathrm{mV} / \mathrm{g}$ for a comb-finger structure of $2-\mu \mathrm{m}$ gap and $5.5 \mathrm{kHz}$ resonant frequency [4].

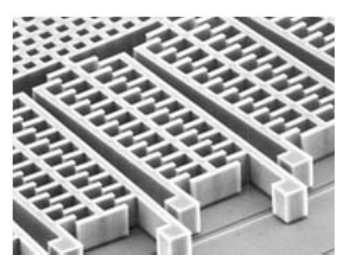

(a)

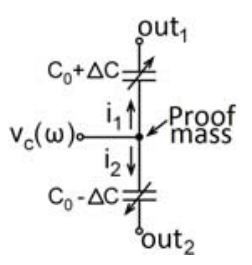

(b)
Fig. 1. a) Surface-micromachined comb-finger capacitive accelerometer (STMicroelectronics) and b) its electrical model.

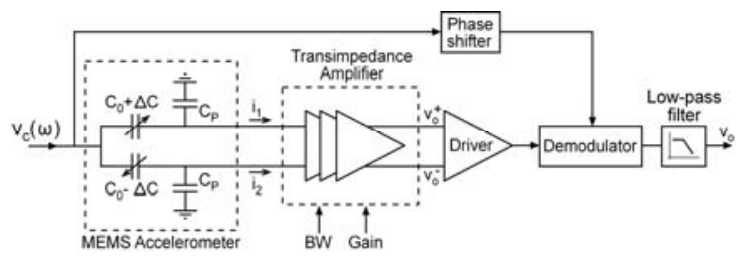

Fig. 2. Conceptual scheme of a capacitance to voltage converter based on transimpedance amplifier and synchronous demodulation. The gain and bandwidth of the transimpedance amplifier are programmed with an externally generated digital word.

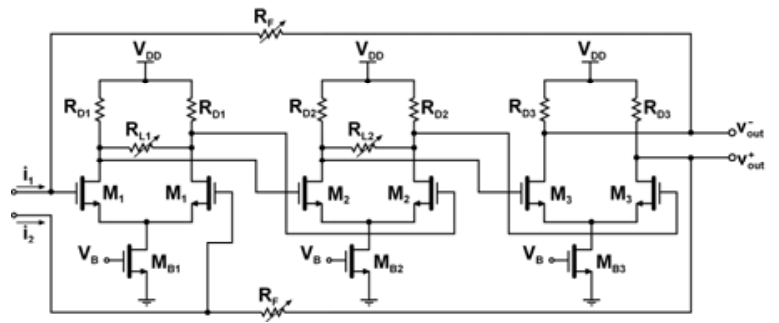

Fig. 3. Schematic circuit of the proposed transimpedance amplifier.

\section{Conclusions}

In this work, a fully-differential transimpedance amplifier with programmable gain and bandwidth has been presented. It has been designed in a 180$\mathrm{nm}$ CMOS technology and it is intended for its use in MEMS accelerometers as a capacitive to voltage converter. Although the proposed TIA is aimed for a differential surface-micromachined comb-finger capacitive accelerometer, its programmability allows its use for other inertial capacitive MEMS sensors.

\section{References}

[1]. KAAJAKARI, V. Practical MEMS, Small Gear Publishing, 2009.

[2]. LÖTTERS, J. C., OLTHUIS, W. VELTINK, P. H., and BERGVELD, P. A sensitive differential capacitance to voltage converter for sensor applications, IEEE Transactions on Instrumentation and Measurement, 1999, 48 (1), 89-96.

[3]. ROYO, G., GIMENO, C., SÁNCHEZ-AZQUETA, C., ALDEA, C., and CELMA, S. CMOS transimpedance amplifier with controllable gain for RF Overlay, Proceedings of $12^{\text {th }}$ Conference on PhD Research in Microelectronics and Electronics, 2016 (accepted).

[4]. ROYO, G., GIMENO, C., ALDEA, C., and CELMA, S. A programmable differential capacitance to voltage converter for MEMS accelerometers, Proceedings of EUROSENSORS XXX, 2016 (pending).

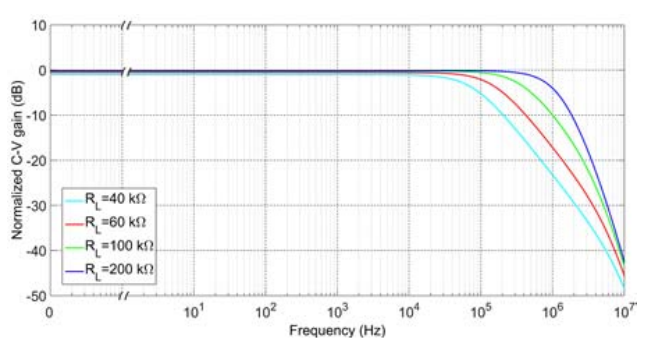

(a)

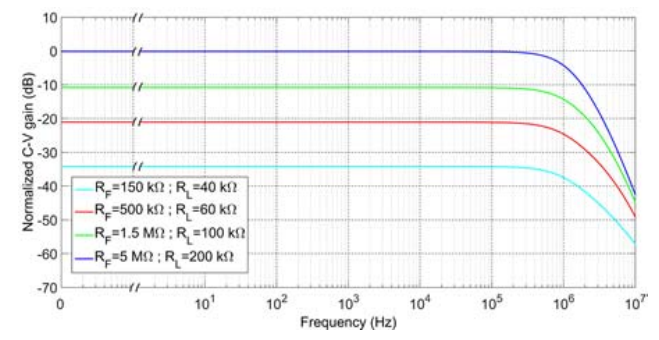

(b)

Fig. 4. Programmable frequency response of the TIA with a) variable bandwidth and b) variable gain. 